\title{
Research Progress on the Relationship Between Interleukins Gene Polymorphism and Type 2 Diabetes
}

\author{
Yang LI ${ }^{a}$, Miao-jing LI $I^{b}$, Xin-yu CUI ${ }^{b}$, Sheng-zhong RONG ${ }^{b}, G e$ \\ $\mathrm{JIN}^{\mathrm{b}}$, Hui ZHANG ${ }^{\mathrm{b}}$, Ying-ying $\mathrm{CHEN}^{\mathrm{b}}$, Qing YE ${ }^{\mathrm{b}}$, Yu-da WANG \\ and Yue GUAN ${ }^{\mathrm{a}, \text { * }}$ \\ ${ }^{a}$ Hongqi Hospital, Affiliated to Mudanjiang Medical University

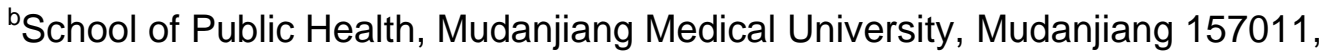 \\ Heilongjiang Province, PR China \\ 91858686@163.com \\ ${ }^{*}$ Corresponding author
}

Keywords: Type 2 Diabetes, Interleukin, Single Nucleotide Polymorphisms.

\begin{abstract}
Inflammation plays a significant role in the etiology and pathogenesis of type 2 diabetes mellitus (T2DM).Many single nucleotide polymorphisms (SNPs) in various genes including inflammatory cytokines have been reported as a risk for T2DM.Interleukins (IL) such as IL - 6, IL - 10, IL - 18 and IL - 1 are strongly associates with increased risk of type 2 diabetes. This review will summarize and recommend studies that investigated associations between Interleukins gene polymorphism and T2DM.
\end{abstract}

\section{Introduction}

With the completion of the Human Genome Project, gene polymorphism (gene polymorphism) has played an important role in clarifying the body's susceptibility to disease, diversity of clinical manifestations of disease and the response to drug treatment. It can diagnose and treat complex disease through the analysis of gene polymorphism and clinical phenotypes, and the relationship between gene polymorphism and the risk of disease. Single nucleotide polymorphism (Single nucleotide polymorphisms, SNPs) refers to a DNA sequence variation at the genomic level caused by a single nucleotide variation, which shall not be less than $1 \%$ of the population frequency distribution [1]. It is by far one of the most common types of human genetic variation accounting for more than $90 \%$ of all known polymorphisms. Those studies have shown that there is a close relationship between interleukins gene polymorphism and T2DM development. In recent years, many scholars studied the relationship of diabetes of interleukins (interleukins, IL) gene polymorphism. Research shows that there were the closely related about interleukin gene polymorphism and T2DM development. This article will state the relationship between T2DM and the gene polymorphism of IL - 6, IL - 10, IL - 18 and IL - 1.

\section{IL - 6 Gene Polymorphism and T2DM}

\section{The Structure and Source of IL - 6}

IL-6 is a multifunctional cytokine which is closely related to cell growth, differentiation and gene expression regulation. It is a kind of biologically active glycoprotein which is encoded by human IL-6 gene. IL-6 is a glycopeptides that the relative molecular mass is 26,000 consisted of 184 amino acid glycoprotein. Human 
IL-6 gene is located on the short arm of chromosome 7, it contains five exons and four introns. IL-6 is inflammatory factors which has the most close relationship with endocrine [2]. IL - 6 derives from a wealth of sources. Nowadays, it has been found that lymphoid cells, endothelial cells, fibroblasts, skeletal muscle and fat cells can produce IL - 6. [3] In basic conditions, IL - 6 in blood is mainly secreted by adipose tissue, it accounts for about $15 \% \sim 35 \%$. In addition, the pancreatic islet B cells can also be autocrine IL - 6. Many factors can stimulate the secretion of IL - 6, such as IL - 1, IL - 2 and INF, PDGF, LPS, insulin, catecholamine, etc. on the contrary, glucocorticoid and sex hormone, IL - 4, IL - 10 can inhibit the expression of IL - 6.

\section{Relationship Between IL-6 and T2DM}

T2DM is a chronic inflammatory condition, as inflammatory cytokines, IL-6 may have involved in this situation. Pickup [4] revealed that the serum of patients with T2DM and IL-6 were non-DM health increases. Leinonen [5] also found that T2DM patients' serum C - RP, IL - 6 and SAA inflammatory markers, such as relatively higher than DM healthy subjects, and all inflammatory markers and negatively correlated with insulin sensitive index. Some scholars study found in patients with severe acute pancreatitis, the concentration of plasma IL - 6 increased significantly, and interleukin 6 in pancreatitis develop into severe acute pancreatitis on the sensitivity of $77.7 \%$ [6], And severe acute pancreatitis patients have different degree of pancreatic B cells is damaged, it also suggests IL - 6 there is a close correlation with the incidence of diabetes.

\section{IL - 18 Gene Polymorphism and T2DM}

\section{The Structure and Source of IL -18}

IL-18 is a kind of newly discovered proinflammatory factor in recent years with the function of multidirectional effect, which play a key role in inflammatory reaction chain. [7] IL -18 expressions in macrophages, dendritic cells, keratinocyte cells, etc. Its main function is the promotion of $\mathrm{T}$ cells and natural killer cells IFN - C, the expression of TNF - A raised IL - 2, IFN - C type Th1 cytokines, promoting the activation of $\mathrm{T}$ cells proliferation [8] As a result of the important role that IL - 18 played in the network. It is associated with the occurrence and development of various diseases. The human IL - 18 genes in 11 q22. 2-22.3, contain six exons and five introns, cDNA total length of about $1.1 \mathrm{~KB}$. Has now been found there are multiple on the IL - 18 gene polymorphism loci, which located in the promoter region, district or exon upstream, transcription promoter region are mainly the $-137,607,132$, while 127,1248 , etc are mainly in exons area.

\section{IL - 18 Relations with T2DM}

Literatures have reported that diabetic patients`serum IL - 18 significantly increased compared with healthy controls. IL -18 is one of the inflammatory cytokines associated with insulin resistance, involved in the occurrence of type 2 diabetes. [9] A number of studies have shown that increasing serum IL - 18 may be associated with the occurrence of T2DM, and the rise of IL - 18 can be used as a sign of T2DM insulin resistance, thus prompting the close relationship between serum levels of IL 18 and T2DM. 


\section{IL - 18 Gene Polymorphism and T2DM}

Giedraitis [10] found that in the IL - 18 gene of exon 2 upstream promoter region that - 137 and - 607 loci exist single nucleotide polymorphisms. So IL-18 gene - 137-607 loci SNP altered these transcription factors and the combining ability of IL-18 gene promoter, which directly affect the expression level of IL -18 and the influence of IFN $-\gamma$ expression and function of Th1 cells [11] One of the studies in han people in zhuhai region shows that IL-18 gene SNPs may be associated with T2DM susceptibility, but it is uncertain whether it is main susceptibility loci of T2DM in China zhuhai regions.

\section{IL - 1 Gene Polymorphism and T2DM}

\section{IL-1 Relations with T2DM}

IL - 1 beta (IL - 1 has a specific injury beta cells) mainly involved in the occurrence of diabetes development [12]. In insulin dependent diabetes animal model, monocyte/macrophage is the main inflammatory cells infiltrating islet. The activation of mononuclear cells via the secretion of IL - 1 beta islet beta cells to specific injury, making beta cell necrosis. The role includes: Firstly significantly inhibit the release of insulin and glucose, stimulate the release of insulin [13]; Secondly trigger beta cells induce NO syntheses (iNOS) expression and strengthen the I - NOS activity [14]: cause most of the cell necrosis, a small number of apoptosis; Thirdly the release of TNF alpha beta cells, thereby selectively destroy beta cells. In patients with T2DM, it can remove off the function of inhibition to Pancreatic islet B cells, IL - 1 in the peripheral blood levels dropped, can make the patients' blood sugar, glycosylated hemoglobin and beta cell function improved [15].

\section{IL - 1 Gene Polymorphism and T2DM}

IL - 1 gene locate on chromosome long arm 2 q13. IL - 1 alpha, beta IL - 1 as the effect of molecular excited agent [16]. In regard to the research of genetic polymorphism of IL-1, ping-an zhang [17] through to the han people, such as IL-1beta in patients with type 2 diabetes, RN gene polymorphism analysis found that although the IL-1beta (511), RN (introns 2) gene polymorphism and susceptibility to type 2 diabetes has no meaning, but IL- $1 \mathrm{RN}$ alleles may be associated with the progression of type 2 diabetes.

\section{Summary and Prospect}

To sum up, there is a close relationship between the occurrence and development between interleukins and diabetes. The changes of some interleukins in the body can indirectly response the immune dynamics of patients with diabetes, which is helpful for the diagnosis, treatment and prognosis of diabetes and it also has great effect on monitoring the occurrence of diabetes and the evaluation of treatment efficacy.

\section{Acknowledgments}

This study was supported by grants from the National Science Foundation of China (No. 81373083,No.81573286), 2014 Young Teachers Project of Heilongjiang Province (No. 1254G062), the Science and Technology Project of Mudanjiang City(Z2014S027; G2015d1843) and Undergraduate Project of Heilongjiang Province (201510229007). 


\section{References}

[1] WangDG, Fan JB, SiaoCJ, et al. Large-scale identification, mapping, and genotyping of single- nucleotide Polymorphisms in the human genome Science, 1998, 280(5366):1077-1082.

[2] Herder C,Peltonen M,Koenig W, et al. Systemic Immune Mediator and lifestyle Changes in the Prevention of Type 2 Diabetes Results From the Finish Diabetes Prevention Study Diabetes 2006,55(8):2340-2346

[3] Snickv,J.linterleukin-

6:Anoverview,AnnuRevmunol.1990,8:253-279.3.S,Akira,.TTaga,T.Kishimoto,Interl eukin-6 in biology and medieine.Ady.Immunol.54(1993):1-78.

[4] Pickup JC, Chu sney GD, Thomas SM, et al Plasma interleukin -6, tumor necrosis factor alpha and blood cytokine production in Type 2 diabetes [J]. Life Sc, 2000, 67: 291.

[5] Leinonen E, Hurt-Camejo E, W iklund O, et al .Insulin resistance and adiposity correlate with acute -phase reaction and soluble cell adhesion molecules in type 2 diabetes[J].A the roscle-rosis, 2003, 166: 387.

[6] SathyanarayanG, GargPK, PrasadH, et al. Elevated level of interleukin -6 predicts organ failure and severe disease in patients with acute pancreatitis $[\mathrm{J}]$. J Gastroenterol Hepato, 1 2007,22(4): 550.

[7] GracieJA,RobertsonSE,MelnnesIB.Interleukin18.JLeukoeBiol.2003Feb:73(2):2 13-214.

[8] Rodriguez-Gal, n MC, Bream JH, Farr A, et al. Synergistic effect of IL-2, IL-12, and IL-18 on thymocyte apoptosis and Th1/Th2 cytokine expression [J]. J Immunology, 2005, 174(5):2796.

[9] Macrophages, Inflammation, and Insulin Resistance. Annual Review of Physiology. 2010, 72: 219-246.

[10] 10.GiedraitisV, HeB, HuangWX, etal. Cloning and mutation analysis of thehumanIL-18Promoter: a possible role of Polymorphisms index Pression regulation. JNeuroimmunol. 2011, 2(2):146-152.

[11] Sugiura T, Kawaguchi Y, Harigai M, et al. Association between adult-onset Stills Disease and interleukin-18 gene Polymorphisms.GenesImmun,2002,3(7):394399

[12] Gao Youliang, Shi Hong, interleukin and diabetes [J], Gansu medicine, 2004, 17 (7): $4-5$

[13] Recent advances in the relationship between obesity, inflammation, and insulin resistance. Eur Cytokine Netw. 2006, 17(1): 4-12.

[14] Anderson AK Sandler S Melatonin protects against strepozotocin ,but not interleukin-1beta-induced damage of rodent pancreatic beta-cells[J].PinealRes.2001,30(3):157-165

[15] Larsen CM, Feuerbach M, Vaasa. et al. Interleukin-1-receptor antagonist in type2 diabetes mellitus [J].N Eng I J Med.2002, 356(15):1517-1526 
[16] Lian Kai, Du Jingyuan. Experimental study on the effect of interleukin 1 on osteoclast induced bone resorption [J]. Chinese Journal of modern medicine, 2001, 11 (100): 6-8.

[17] Mechanisms linking obesity to insulin resistance and type 2 diabetes. Nature $2006,444,840-846$. 